\title{
„Mit gleichen Wahlmöglichkeiten in der Gemeinschaft leben“- Behinderungen und Enthinderungen selbstbestimmter Lebensführung
}

\author{
Gudrun Wansing
}

$\begin{array}{lr}\text { I. Einleitung } & 69\end{array}$

$\begin{array}{ll}\text { II. Inklusion, Autonomie und Lebenslagen } & 71\end{array}$

III. Inklusionsrückstände und verspätete Befreiung von Menschen mit Beeinträchtigungen

IV. Mit gleichen Wahlmöglichkeiten in der Gemeinschaft leben 75

V. Strukturelle Entwicklungen der wohnbezogenen Unterstützung 77

$\begin{array}{ll}\text { VI. Bewertungen und Wünsche aus Nutzerperspektive } & 79\end{array}$

VII. Fazit und Perspektiven der Enthinderung $\quad 82$

$\begin{array}{ll}\text { VIII. Literatur } & 85\end{array}$

\section{Einleitung}

Im Mittelpunkt des folgenden Beitrages steht Artikel 19 der UN-Behindertenrechtskonvention (im Folgenden BRK), dessen Leitperspektiven ,unabhängige Lebensführung und Einbeziehung in die Gemeinschaft (independent living and inclusion in community)“ genuin auf eine kommunale bzw. Sozialraumorientierung ausgerichtet sind.

Art. 19 Unabhängige Lebensführung und Einbeziehung in die Gemeinschaft

Die Vertragsstaaten dieses Übereinkommens anerkennen das gleiche Recht aller Menschen mit Behinderungen, mit gleichen Wahlmöglichkeiten wie andere Menschen in der Gemeinschaft zu leben, und treffen wirksame und geeignete Maßnahmen, um Menschen mit Behinderungen den vollen Genuss dieses Rechts und ihre volle Einbeziehung in die Gemeinschaft und Teilhabe an der Gemeinschaft zu erleichtern, indem sie unter anderem gewährleisten, dass

a) Menschen mit Behinderungen gleichberechtigt die Möglichkeit haben, ihren Aufenthaltsort zu wählen und zu entscheiden, wo und mit wem sie leben, und nicht verpflichtet sind, in besonderen Wohnformen zu leben; 
b) Menschen mit Behinderungen Zugang zu einer Reihe von gemeindenahen Unterstützungsdiensten zu Hause und in Einrichtungen sowie zu sonstigen gemeindenahen Unterstützungsdiensten haben, einschließlich der persönlichen Assistenz, die zur Unterstützung des Lebens in der Gemeinschaft und der Einbeziehung in die Gemeinschaft sowie zur Verhinderung von Isolation und Absonderung von der Gemeinschaft notwendig ist;

c) gemeindenahe Dienstleistungen und Einrichtungen für die Allgemeinheit Menschen mit Behinderungen auf der Grundlage der Gleichberechtigung zur Verfügung stehen und ihren Bedürfnissen Rechnung tragen.

Die folgenden Ausführungen beleuchten die Bedeutung dieses Artikels aus sozialwissenschaftlicher Perspektive ${ }^{1}$ zum einen im Kontext konzeptioneller Entwicklungen von Inklusion, Autonomie und Lebenslagen und zum anderen im Horizont empirischer Befunde zur Wohnsituation von Menschen mit Beeinträchtigungen ${ }^{2}$. Im Fokus der Ausführungen stehen Personen, die im Zusammenhang mit erheblichen, teils mehrfachen körperlichen, geistigen oder seelischen Beeinträchtigungen auf umfängliche Unterstützung zur Bewältigung ihres Alltags angewiesen sind (z.B. in den Bereichen Mobilität, Selbstversorgung, Kommunikation und soziale Beziehungen). Ihre Lebensführung bleibt in vielen Aspekten - zuweilen ein Leben lang - geprägt durch die Ambivalenz von Autonomie und Angewiesenheit. Diese Situation wird sich für viele Personen auch durch eine möglicherweise inklusive, barrierefreie Umweltgestaltung zukünftig nicht vollständig auflösen lassen, vielmehr konstituiert sich Behinderung in vielen Lebenssituationen gerade durch ein „Mehr an sozialer Abhängigkeit“3. Dieser Widersprüchlichkeit ist in einer ausgewogenen Balance zu begegnen, wie sie Rappaport bereits für das sozialpolitische Konzept des ,empowerment“ formuliert hatte. Demnach „,können wir nicht länger Menschen als ,Kinder in Not' oder als ,Bürger mit Rechten“ sehen, sondern vielmehr als vollwertige menschliche Wesen, die sowohl Rechte als auch Bedürfnisse haben" $" 4$.

1 Für eine rechtliche Kommentierung von Artikel 19 vgl. Banafsche, Art. 19 Unabhängige Lebensführung und Einbeziehung in die Gemeinschaft, in: Welke, UN-Behindertenrechtskonvention mit rechtlichen Erläuterungen, Berlin 2012, S. 150-163.

2 Im Folgenden wird weitgehend zwischen den Begriffen Beeinträchtigung (im Sinne funktionaler Einschränkungen) und Behinderung als sozialer Prozess und als Ergebnis der negativen Wechselwirkungen von Beeinträchtigungen und Kontextfaktoren unterschieden (vgl. die dynamischen und interaktiven Konzepte von Behinderung in der ICF und der BRK).

3 Hahn, Behinderung als soziale Abhängigkeit. Zur Situation schwerbehinderter Menschen, München 1981.

4 Rappaport, Ein Plädoyer für die Widersprüchlichkeit: Ein sozialpolitisches Konzept des „empowerment“ anstelle präventiver Ansätze, Verhaltenstherapie und psychosoziale Praxis 1985, S. 267. 


\section{Inklusion, Autonomie und Lebenslagen}

Artikel 19 greift mit der Formulierung ,unabhängige Lebensführung ${ }^{5}$ und Einbeziehung in die Gemeinschaft" die untrennbare Verknüpfung von Autonomie und Inklusion auf. Bereits in der Präambel der BRK wird die Erkenntnis formuliert, „wie wichtig die individuelle Autonomie und Unabhängigkeit für Menschen mit Behinderungen ist, einschließlich der Freiheit, eigene Entscheidungen zu treffen“ (Präambel, n). Ausgehend von gesellschaftlichen Modernisierungsprozessen wie der Freisetzung des Menschen aus vorgegebenen sozialen Mustern, der Individualisierung und der Pluralisierung von Lebensstilen ist das Recht auf Selbstbestimmung ein konstitutives Moment der Teilhabe an den kulturellen Errungenschaften einer demokratisch verfassten Gesellschaft.

Die Freiheit des Individuums steht keineswegs im Widerspruch zum Grundsatz der sozialen Einbeziehung (Inklusion); vielmehr stehen Autonomie und Inklusion in einer unmittelbaren Wechselwirkung zueinander. „Erst in der wechselseitigen Verwiesenheit wird klar, dass Autonomie gerade nicht die Selbstmächtigkeit des ganz auf sich gestellten Einzelnen (...) meint, sondern auf selbstbestimmtes Leben in sozialen Bezügen zielt; und im Gegenzug wird deutlich, dass soziale Inklusion ihre Qualität gerade dadurch gewinnt, dass sie Raum und Rückhalt für persönliche Lebensgestaltung bietet" ${ }^{\text {"6 }}$. Ein Leben nach eigenen Vorstellungen und Zielen führen zu können, setzt voraus, Zugang zu den verschiedenen Gesellschaftssystemen (wie Bildung, Arbeitsmarkt, Gesundheit) zu haben und (von diesen) mit relevanten Zutrittsressourcen (wie Bildungsabschlüssen, Geld, Netzwerken, politischer Macht) ausgestattet zu werden. Inklusion zielt also auf die gesellschaftlichen Voraussetzungen für soziale Teilhabe, und Autonomie bedeutet, diese strukturellen Chancen gemäß den eigenen, individuellen Lebensplänen verwirklichen zu können. In diesem Rahmen können Selbstbestimmung und Identität auch über Nicht-Zugehörigkeit und Nicht-Partizipation zum Ausdruck kommen, sofern diese selbstgewählt sind. Allerdings fallen die Freiheitsgrade der Teilhabe in den verschiedenen Gesellschaftssystemen unterschiedlich aus. So ist es in westlichen, demokratischen Gesellschaften eine höchst freiwillige Entscheidung, eine Religionszugehörigkeit zu haben oder sich für Kunst oder Sport zu interessieren. Hingegen sind die Partizipation an Bildung (über die Schulpflicht) und die Teilhabe an Erwerbsarbeit soziale Erwartungen, denen sich niemand entziehen kann. Der Zugang zu diesen Bereichen und

5 Vor dem Hintergrund der dargestellten Ambivalenz von Autonomie und Abhängigkeit wäre der Schattenübersetzung der BRK Vorzug zu geben, die den Begriff „Unabhängigkeit“ durch den Begriff „Selbstbestimmung“ ersetzt: „Selbstbestimmt Leben und Einbeziehung in die Gemeinschaft“, vgl. http://www.netzwerk-artikel-3.de/attachments/093_schattenuebersetzung-endgs.pdf.

6 Bielefeldt, Zum Innovationspotenzial der UN-Behindertenrechtskonvention. Deutsches Institut für Menschenrechte, 3. Aufl. Berlin 2009, S. 11. 
die erfolgreiche Teilhabe, im Sinne der Ausstattung mit Ressourcen, sind Voraussetzung für soziale Zugehörigkeit und Anerkennung insgesamt ${ }^{7}$.

Die wechselseitige Verbindung von Inklusion und Autonomie in der BRK liefert konzeptionelle wie empirische Anschlussstellen zum Lebenslagenansatz, der gegenwärtig (erneut) sowohl in den fachlichen Diskursen zu Behinderung und Teilhabe ${ }^{8}$ als auch für Sozialpolitik und Sozialberichterstattung 9 eine wichtige Rolle spielt. Nach Weisser, der den Lebenslagenansatz bereits in den 1950er Jahren in die Sozialpolitik eingebracht hatte, ist Lebenslage „der Spielraum, den die äußeren Umstände dem Menschen für die Erfüllung der Grundanliegen bieten, die er bei unbehinderter und gründlicher Selbstbesinnung als bestimmend für den Sinn seines Lebens ansieht" 10 . In der neueren Auseinandersetzung mit dem Lebenslagenansatz werden Parallelen zum Konzept der Verwirklichungschancen (,capability approach“) von Amartya Sen diskutiert, das internationale Aufmerksamkeit erfährt ${ }^{11}$. Auch Sen betrachtet Wahlfreiheiten im Sinne von „Möglichkeiten oder umfassenden Fähigkeiten („,capabilities“) von Menschen, ein Leben führen zu können, für das sie sich mit guten Gründen entscheiden konnten und das die Grundlagen der Selbstachtung nicht in Frage stellt"12, als bedeutsam für das Wohlergehen. Gemeinsam ist dem Lebenslagenansatz, in seinen durchaus unterschiedlichen Akzentuierungen, und dem Ansatz der Verwirklichungschancen eine mehrdimensionale Perspektive auf Lebensbedingungen, welche objektive Voraussetzungen (Handlungsspielräume) und subjektive Wahrnehmungs-, Deutungs- und Bewältigungsmuster (Präferenzen, Selbstbestimmung) miteinander verbindet. Ein solcher Betrachtungsrahmen erweist sich als zielführend für eine Bewertung der Lebenssituation beeinträchtigter Menschen im Lichte der BRK und hierauf bezogene sozialpolitische Interventionen.

7 Wansing, Inklusion in einer exklusiven Gesellschaft - oder: wie der Arbeitsmarkt Teilhabe behindert, Behindertenpädagogik 2012, S. 381-396.

8 Beck/Greving, Lebenslage und Lebensbewältigung. Enzyklopädisches Handbuch der Behindertenpädagogik 5, Stuttgart 2012.

9 Engels, Lebenslagen, in: Maelicke (Hrsg.), Lexikon der Sozialwirtschaft, Baden Baden 2008, S. 643-646. Hornberg/Schröttle, Vorstudie zur Neukonzeption des Behindertenberichts, in: Bundesministerium für Arbeit und Soziales (Hrsg.), Forschungsbericht 408/2012, http://www.bmas.de/ DE/Service/Publikationen/Forschungsberichte/Forschungsberichte- Teilhabe/ $\mathrm{fb}$-fb408-vorstudie-zur-neukonzeption-des-behindertenberichtes.html.

10 Leßmann, Lebenslagen und Verwirklichungschancen (capability) - Verschiedene Wurzeln, ähnliche Konzepte, Vierteljahresschrift zur Wirtschaftsforschung 75, 2006, S. 33.

11 Ebd.

12 Sen, Ökonomie für den Menschen. Wege zur Gerechtigkeit und Solidarität in der Marktwirtschaft, München, Wien 2000, S. 29. 


\section{Inklusionsrückstände und verspätete Befreiung von Menschen mit Be- einträchtigungen}

Erst seit dem Übergang zum 21. Jahrhundert gelangt (in Deutschland) verstärkt ins politische Bewusstsein, dass die Lebenslagen von Menschen mit Beeinträchtigungen vielfach durch Missachtung ihrer Rechte und mehrdimensionale Risiken sozialer Benachteiligung und Ausgrenzung geprägt sind ${ }^{13}$. Mit vielen Jahrzehnten Verspätung gemessen an der allgemeinen Inklusionsdynamik moderner Wohlfahrtsstaaten im Zuge der Ausweitung bürgerlicher, politischer und sozialer Rechte und ihres „Postulats einer Vollinklusion aller Menschen" 14 - wurden sie überhaupt erst als Bürgerinnen und Bürger mit gleichen Rechten wahrgenommen ${ }^{15}$. Und erst im Zuge der Anerkennung ihrer uneingeschränkten Ansprüche auf Inklusion, auf soziale Zugehörigkeit, auf Selbstbestimmung und soziale Teilhabe werden faktische „Inklusionsrückstände“ 16 in Form von Benachteiligung und sozialer Ausgrenzung, Diskriminierung und Fremdbestimmung, oder anders formuliert: Behinderungen ihrer sozialen Teilhabe, überhaupt erst wahrnehmbar und als mögliche Verletzung von Rechten begründungsbedürftig. Zuvor war man über einen sehr langen Zeitraum davon ausgegangen, dass Behinderung ein individuelles Schicksal bzw. ein persönliches Problem von Menschen ist, die aufgrund von Vererbung, Krankheit oder Unfall körperlich, seelisch oder geistig von einer ,normalen Gesamtbevölkerung abweichen. Soziale Benachteiligungen der Betroffenen wurden in diesem stark biologisch geprägten Erklärungsmodell als unabänderliche Folge ihrer individuellen Defizite gesehen. Interventionen richteten sich entsprechend auf einzelne Personen und ihre Therapie, Förderung und Betreuung bzw. auf ihre Verwahrung und Versorgung in abgesonderten Lebensräumen. Kontextfaktoren blieben dabei weitgehend unberücksichtigt. Wichtige Impulse für eine neue, rechtebasierte und auf die soziale Dimension fokussierte Sichtweise auf Behinderung gingen seit den 1970er, 80er Jahren wesentlich von der organisierten Behindertenbewegung und ihrer Forderung nach Selbstbestimmung, Selbstvertretung und der Abschaffung von Sondereinrichtungen aus. Sie machte darauf aufmerksam, dass die benachteiligte Lebenssituation von Menschen mit Beeinträchtigungen nicht naturgegeben und keine unabänderliche Folge persönlicher Defizite ist, sondern wesentlich durch soziale Faktoren bedingt ist, welche sie an

13 Für einen internationalen Vergleich siehe WHO, World Report on Disability, Genf 2011.

14 Luhmann, Die Gesellschaft der Gesellschaft, Bd. 2, Frankfurt a.M. 1999, S. 630.

15 Auf der Linie „der evolutionären Dynamik moderner Gesellschaften“ (Burzan, Das Publikum der Gesellschaft. Inklusionsverhältnisse und Inklusionsprofile in Deutschland, Wiesbaden 2008, S. 27), die zu einer Ausweitung der Zugangsrechte und ihrer faktischen Umsetzung führt, lassen sich auch die amerikanische Bürgerrechtsbewegung der Schwarzen in den 50er und 60er Jahren sowie die Emanzipationsbewegung der Frauen in den 70er Jahren des 21. Jahrhunderts in Anlehnung an Parsons als ,nachholende Modernisierung“ beschreiben (ebd.).

16 Burzan (Fußn. 15), S. 27. 
der Ausübung ihrer Grundrechte wie Freiheit, Privatheit und Selbstbestimmung behindern.

Diese gesellschaftspolitische Dimension von Behinderung und Teilhabe wurde jedoch in der Entwicklung der deutschen Behindertenhilfe lange Zeit - und wird es zum Teil noch heute - ausgeblendet. Dabei waren wichtige politische und fachliche Impulse bereits seit den 1950er Jahren vom Normalisierungsprinzip ausgegangen, das im Kontext skandinavischer Sozialpolitik entwickelt worden war. Vor dem Hintergrund der scharfen Kritik am biologistischen Menschenbild und der Anstaltsverwahrung mit ihren menschunwürdigen Lebensbedingungen, insbesondere für Menschen mit geistiger Behinderung, folgte man hier dem Grundsatz „to create existence for the mentally retarded as close to normal living conditions as possible"17. Es ist interessant, sich die Formulierungen des Juristen und Verwaltungsbeamten Bank-Mikkelsen (der das Normalisierungsprinzip in die dänische Sozialgesetzgebung eingebracht hatte) im Lichte der BRK noch einmal im Wortlaut anzuschauen: „This is normalization; equality with other citizens without categorizing groups"18. Mit diesem politischen Richtungswechsel von besonderen Programmen für als ,behindert' bezeichnete Bevölkerungsgruppen hin zur Gewährleistung gleichberechtigter Lebensbedingungen für alle Bürgerinnen und Bürger wurden wichtige Grundsätze der BRK bereits vorweggenommen. Während die Leitperspektive Normalisierung jedoch in Skandinavien in Verbindung mit der dortigen Bürgerrechtstradition nachhaltige sozialpolitische Reformen bis hin zur Auflösung von Sondereinrichtungen nach sich zog, blieb die Umsetzung in Deutschland im Wesentlichen auf die Weiterentwicklung des professionellen Hilfesystems und seiner Institutionen beschränkt ${ }^{19}$. Dabei war die Neugestaltung im Wesentlichen durch fachliche Vorstellungen von ,normalen` Lebensbedingungen geleitet. Standards für Wohnqualität wurden vielfach ohne die wirksame Partizipation ${ }^{20}$ der Menschen mit Beeinträchtigungen entwickelt und richteten sich an einer unterstellten Homogenität einer Gruppe der Behinderten und an deren vermeintlich kollektiven Unterstützungsbedarfen aus.

Erst im Zuge einer „verspäteten Befreiung“21 von Menschen mit Beeinträchtigungen und dem Einzug der Leitlinie Selbstbestimmung in die Unterstützungssysteme der Be-

17 Bank-Mikkelsen, in: Flynn/Nitsch (Hrsg.), Normalization, social integration, and community services, Baltimore 1980, S. 51-70.

18 Bank-Mikkelsen (Fußn. 17), S. 62.

19 Gleichwohl zum Beispiel Thimm auch für Deutschland grundlegende Reformansätze für eine gemeindeorientierte Behindertenhilfe formuliert hatte: Thimm, Leben in Nachbarschaften. Hilfen für Menschen mit Behinderungen, Freiburg 1994; ders., Tendenzen gemeinwesenorientierter Hilfen Gesellschaftliche Ausrichtungen und fachliche Konsequenzen, in: ders. (Hrsg.), Das Normalisierungsprinzip. Ein Lesebuch zu Geschichte und Gegenwart eines Reformkonzeptes, Marburg 2005.

20 Als wirksam erweist sich praktizierte Partizipation dann, wenn sich die subjektiven Erfahrungen, Interessen und Wünsche der beeinträchtigten Menschen auch faktisch auf die Gestaltung der Wohnund Lebensbedingungen auswirken und sich ihre Mitwirkung nicht in einer formalen Scheinbeteiligung bzw. bloße physische Anwesenheit in Planungsprozessen erschöpft.

21 Waldschmidt, Selbstbestimmung als Konstruktion. Alltagstheorien behinderter Frauen und Männer, 2. Aufl. Wiesbaden 2012, S. $32 \mathrm{ff}$. 
hindertenhilfe zu Beginn der 1990er Jahre wurde die subjektive Perspektive der Adressaten deutlich gestärkt. Neue Methoden der individuellen Hilfeplanung wurden eingeführt, die Position von Heimbeiräten gestärkt und Nutzerzufriedenheit avancierte zu einem wichtigen Indikator von Ergebnisqualität der sozialen Einrichtungen und Dienste. Dennoch blieben und bleiben die Grundsätze der Autonomie und Partizipation, solange sie ausschließlich im Rahmen organisierter, professioneller Dienstleistungen betrachtet werden, auf die Rolle des Konsumenten bzw. des Nutzers ${ }^{22}$ reduziert. Handlungsspielräume für Selbstbestimmung und Teilhabe werden durch eine vorgegebene Versorgungsstruktur abgesteckt und enden (bildlich gesprochen) an den Grundstücksgrenzen von Einrichtungen. So aber bleiben Optionen und Anforderungen einer kulturell üblichen und anerkannten Lebensführung begrenzt. An dieser Stelle nun liefert die UNKonvention weiterführende Impulse.

\section{Mit gleichen Wahlmöglichkeiten in der Gemeinschaft leben}

Artikel 19 der Behindertenrechtskonvention formuliert als Maßstab für die Verwirklichung einer selbstbestimmten Lebensführung „das gleiche Recht aller Menschen mit Behinderungen, mit gleichen Wahlmöglichkeiten wie andere Menschen in der Gemeinschaft zu leben“. Während im Rahmen der allgemeinen Grundsätze der BRK die abstrakte Makroebene der Gesamtgesellschaft und ihre unterschiedlichen Funktionssysteme (wie Bildung, Arbeit und Recht) als Referenzrahmen für Inklusion (,inclusion in society“, Art. 3) zu betrachten sind ${ }^{23}$, steckt hier die „,community“ (Gemeinschaft, Gemeinde) als konkreter, örtlicher und sozialer Erwartungs- und Erfahrungsraum alltäglicher Lebensführung den Betrachtungsrahmen ab. „Auf der Ebene einer Region ist der soziale Raum öffentlicher und politischer Raum der Interessensvertretung, der Sozialplanung, der Infrastrukturen, des bürgerschaftlichen Engagements, der Kultur und vieles mehr; er ist Ort der Lebensbewältigung von Menschen (Alltagsbewältigung) und räumliche Dimension ihrer sozialen Beziehungen (soziale Netzwerke), er bildet die Vermittlungsinstanz zwischen Individuum und Gesellschaft als Ort der Vergemeinschaftung, als Bürger, in Vereinen, Nachbarschaften, Freundschaftsbeziehungen“24. Einbeziehung in die Gemeinschaft meint den gleichberechtigten Zugang zu allen Aktivitäts-, Partizipationsund Kontaktspielräumen am (selbstgewählten) Wohn- und Lebensort. Die Formulierung „mit gleichen Wahlmöglichkeiten wie andere Menschen“ wirft jedoch insofern Schwie-

22 Schäfers, Lebensqualität aus Nutzersicht. Wie Menschen mit geistiger Behinderung ihre Lebenssituation beurteilen, Wiesbaden 2008; Schlebrowski, Starke Nutzer im Heim. Wirkung persönlicher Budgets auf soziale Dienstleistungen, Wiesbaden 2009.

23 Wansing, Der Inklusionsbegriff in der Behindertenrechtskonvention, in: Welke (Hrsg.), Behindertenrechtskonvention und rechtliche Erläuterungen, Berlin 2012, S. 93-103.

24 Beck/Greving, Lebenswelt, Lebenslage, in: dies. (Hrsg.), Lebenslage und Lebensbewältigung. Enzyklopädisches Handbuch der Behindertenpädagogik 5, Stuttgart 2012, S. 50. 
rigkeiten auf, als sie zum einen die Möglichkeit einer eindeutigen Differenzierung zwischen Menschen mit Beeinträchtigungen und anderen Menschen unterstellt und zum anderen von gleichen Zugangschancen der ,anderen“ ausgeht. Faktisch zeigen sich aber in der Bevölkerung insgesamt höchst ungleiche Wahlmöglichkeiten in der Gemeinschaft (etwa im Zugang zu Sportvereinen oder zum Ehrenamt) in Abhängigkeit von Geschlecht, Alter, Ethnizität, Klasse oder Schicht. Zugleich sind diese Ungleichheiten auch in der Gruppe der Menschen mit Beeinträchtigungen ausgebildet. ${ }^{25}$ Diese Schwierigkeit, soziale Gruppen als Maßstab für gleiche Wahlmöglichkeiten zu bilden, wird in der BRK dadurch umgangen, dass die Entscheidungsspielräume in Artikel 19 a-c inhaltlich bestimmt werden, und zwar als a) freie Wahl des Aufenthaltsortes und der Wohnform, b) Zugang zu gemeindenahen Unterstützungsdiensten und c) Verfügbarkeit bedürfnisgerechter gemeindenaher Dienste und Einrichtungen. ${ }^{26}$ Damit werden Optionen für Selbstbestimmung und Teilhabe formuliert, die weit über bislang in Deutschland praktizierte Bewertungsmaßstäbe betreuter Wohnformen hinaus reichen, insofern hier gleiche Bedingungen im Gemeinwesen den Horizont des Möglichen und des Nötigen umreißen. Über die hier formulierten Rechte und Pflichten erfährt auch die Zielperspektive der Leistungen zur Teilhabe am Leben in der Gemeinschaft nach § 5 SGB IX eine inhaltliche Konkretisierung 27.

Zukünftige Sozialforschung und Sozialberichterstattung zu den Lebenslagen von Menschen mit Beeinträchtigungen in der Dimension alltäglicher Lebensführung und hierauf basierende regionale/örtliche Sozialplanungen werden sich an diesen Maßstäben der BRK ausrichten müssen. Hierzu bedarf es entsprechend veränderter methodologischer und methodischer Zugänge. Bislang liegen jedoch kaum aussagekräftige Daten vor, die Auskunft darüber geben könnten, inwieweit und unter welchen (persönlichen und örtlichen) Bedingungen der Anspruch auf unabhängige Lebensführung und Einbeziehung in die Gemeinschaft eingelöst oder seine Verwirklichung behindert wird. So gibt es keine systematischen und verlässlichen Informationen über die (regionale) Verfügbarkeit und Zugänglichkeit bedarfs- und bedürfnisgerechter, gemeindenaher Unterstützungsdienste. Auch fehlen repräsentative Daten zu Wahlmöglichkeiten bezüglich des Wohnortes und der Wohnform sowie zur subjektiven Bewertung von Möglichkeiten der Selbstbestimmung, der sozialen Einbindung und der Partizipation im Gemeinwesen seitens beeinträchtigter Menschen.

In Anbetracht dieser unzureichenden Datenlage lassen sich mögliche Missstände und Entwicklungserfordernisse gegenwärtig nur über empirische Hilfskonstruktionen ableiten. Wichtige Hinweise über Barrieren und Behinderungen der selbstbestimmten Le-

25 Hier zeigt sich die Notwendigkeit, in Forschung und Theoriebildung tradierte Differenzkategorien und ihre (Wechsel-)Wirkungen zu hinterfragen, vgl. Wansing/Westphal, Teilhabeforschung, Disability Studies und Migrationsforschung verbinden. Konzepte von Behinderung und Migration in der Forschung, Orientierung 2012, S. 12-15.

26 Vgl. hierzu auch Banafsche (Fußn. 1), S. 152 f.

27 Banafsche (Fußn. 1), S. $151 \mathrm{f}$. 
bensführung und der Einbeziehung in die Gemeinschaft liefern verfügbare Strukturdaten zur Inanspruchnahme und zu Angebotsformen wohnbezogener Hilfen ebenso wie vorliegende einzelne (regionale und qualitative) Studien zur Bewertung der Wohnsituation durch Menschen mit Beeinträchtigungen. Insgesamt können Wohninstitutionen als „zeitgebundene und insofern kulturell codierte Repräsentationen eines wandelbaren Begriffs von Behinderung" 28 als Seismograph für den Stand der Umsetzung der BRK (im Ausschnitt von Artikel 19) betrachtet werden.

\section{Strukturelle Entwicklungen der wohnbezogenen Unterstützung}

Derzeit gibt es bundesweit insgesamt etwa 340.000 Menschen mit einer amtlich anerkannten Behinderung, die wohnbezogene Leistungen der Eingliederungshilfe ${ }^{29}$ in Anspruch nehmen ${ }^{30}$. Dabei zeigt sich in den letzten zehn Jahren eine ungebrochen steigende Nachfrage ${ }^{31}$, deren Gründe vielfältig und regional unterschiedlich sind. Eine wichtige Rolle spielen eine höhere Lebenserwartung von Menschen mit (kognitiven) Beeinträchtigungen sowie eine zunehmende Nachfrage nach Unterstützung von Menschen mit psychischer Erkrankung. Obwohl es inzwischen zum Teil recht differenzierte Wohnformen für Menschen mit Beeinträchtigungen gibt, weisen die verfügbaren Statistiken keine differenzierten Angaben zur Art der in Anspruch genommenen wohnbezogenen Hilfen aus. Es wird lediglich zwischen stationär (in Wohneinrichtungen) und ambulant (eigene Wohnung oder Wohngemeinschaft) unterschieden. Dabei weisen diese Zahlen auf hohe Zuwachsraten im ambulanten Bereich bei gleichzeitig geringen Steigerungszahlen bei den stationären Leistungsempfängern hin. Gleichwohl zeigt sich, dass der Grundsatz ambulant vor stationär (gemäß § 13 SGB XII, Abs. 1) noch nicht konsequent umgesetzt wird und stationäre Wohnformen nach wie vor dominieren. Im bundesweiten Durchschnitt zeigt sich für das Jahr 2010 ein Verhältnis stationär zu ambulant von knapp zwei Drittel zu einem Drittel ${ }^{32}$. Dabei sind die Chancen der Wahlfreiheit regional höchst ungleich verteilt: In einigen Bundesländern ist die Ambulantisierung der wohnbezogenen Hilfen und der Ausbau differenzierter Unterstützungsangebote bereits deutlich vorangeschritten (z.B. Hamburg, NRW). In anderen Ländern (z.B. SachsenAnhalt, Bayern) hingegen liegt die sog. Ambulantisierungsquote deutlich unter dem

28 Rudloff, Das Ende der Anstalt?, in: Bösl/Klein/Waldschmidt (Hrsg.), Disability History. Konstruktionen von Behinderung in der Geschichte. Eine Einführung, Bielefeld 2010, S. 186.

29 Hilfen zu einem selbstbestimmten Lebens in betreuten Wohnmöglichkeiten nach § 53 SGB XII.

30 Statistisches Bundesamt, Ergebnisse der Sozialhilfestatistik 2010, Wiesbaden 2012, www.destatis. de/DE/Publikationen/WirtschaftStatistik/Sozialleistungen/ErgebnisseSozialhilfe2010_032012.pdf? blob=publicationFile.

31 Vgl. Sozialhilfestatistiken des Statistischen Bundesamtes.

32 Statistisches Bundesamt (Fußn. 30). 
Bundesdurchschnitt ${ }^{33}$ und es gibt zum Teil erhebliche Angebotslücken; dies gilt vor allem in ländlichen Regionen. Zudem sind Wahlmöglichkeiten ungleich verteilt in Abhängigkeit von der Art der Beeinträchtigung bzw. von Art und Umfang der erforderlichen Unterstützung. So sind es insbesondere Menschen mit psychischen Beeinträchtigungen, die zahlenmäßig am deutlichsten von den neuen, offenen Hilfen ${ }^{34}$ profitieren, während es für Menschen (mit kognitiven und mehrfachen Beeinträchtigungen), die auf umfassende, psychosoziale Unterstützung und Pflege bei der Alltagsbewältigung angewiesen sind, nach wie vor wenig Alternativen gibt zwischen einem Leben in einem Wohnheim oder dem Verbleib in der Herkunftsfamilie. Auf der Basis regionaler Studien und Expertenschätzungen kann man davon ausgehen, dass etwa die Hälfte der Menschen mit kognitiven Beeinträchtigungen auch noch im fortgeschrittenen Erwachsenenalter in der Herkunftsfamilie lebt ${ }^{35}$. Die erforderliche Unterstützung und Pflege wird in diesem Rahmen häufig ausschließlich durch Angehörige und ohne Inanspruchnahme professioneller Dienste erbracht. Auch wenn diese enorme Leistungsbereitschaft der Familien Anerkennung verdient, so wäre doch auch zu hinterfragen, inwieweit sich ein langer Verbleib in der Familie nicht im Einzelfall kontraproduktiv zur Perspektive einer selbstbestimmten Lebensführung und der Einbeziehung in das Gemeinwesen auswirken könnte. In jedem Fall gilt es aber, die Situation der Familien in sozialplanerischen Überlegungen zur Unterstützung im Gemeinwesen künftig stärker zu berücksichtigen.

Die uneingeschränkte Verwirklichung der Option, entscheiden zu können, wo und mit wem man leben möchte, und nicht verpflichtet zu sein, in besonderen Wohnformen zu leben (Art. 19, a BRK), wird für Menschen mit Beeinträchtigungen gegenwärtig strukturell durch fehlende Angebote sowie durch den Mehrkostenvorbehalt in den rechtlichen Bestimmungen der Eingliederungshilfe verhindert. Demnach gilt der gesetzliche Vorrang ambulanter Leistung nicht, „wenn eine Leistung für eine geeignete stationäre Einrichtung zumutbar und eine ambulante Leistung mit unverhältnismäßigen Mehrkosten verbunden ist“" (§ 13 SGB XII). Diese Regelung schwächt faktisch die Wirksamkeit des allgemeinen Wunsch- und Wahlrechtes im SGB IX und widerspricht dem Menschenrecht auf freie Wahl der Wohnform. „Im Lichte von Art. 19 des Übereinkommens der Vereinten Nationen (...) kann eine unfreiwillige Heimunterbringung grundsätzlich nicht mehr als zumutbar gelten" 36 . Insbesondere für Menschen mit umfänglichen und damit kostenintensiven Unterstützungsbedarfen bedeutet der Kostenvorbehalt eine er-

33 BAGüS, con_sens, Kennzahlenvergleich der überörtlichen Träger der Sozialhilfe 2010, Hamburg 2011, http://www.consens-info.de/upload/files/projekte/z_projekte_dateien_117219_ueoeTr_ Benchmarking_Bericht_2010_Endfassung.pdf.

34 Gemeint ist die Gesamtheit der ambulanten, personenbezogenen sozialen Dienstleistungen, einschließlich der Persönlichen Assistenz.

35 Für die Region Westfalen-Lippe vgl. Schäfers/Wansing, Familienunterstützende Hilfen (FUH). Alternativen zum betreuten Wohnen für Menschen mit Behinderung, Münster 2009, www.lwl.org/ spur-download/fuh/fuh-bericht.pdf.

36 Welti, Kommentar zu $§ 9$ SGB IX, in: Lachwitz/Schellhorn/Welti (Hrsg.), HK-SGB IX, 3. Aufl. Köln 2010, S. 107. 
hebliche Einschränkung ihres Selbstbestimmungsrechtes. Ein Leben in selbstgewählten Wohnformen am selbstgewählten Wohnort bleibt im Kontext dieser Vorschrift eine exklusive Möglichkeit, die ausschließlich einem unter ökonomischen Gesichtspunkten ausgewählten Personenkreis gewährt wird. Solange am grundsätzlichen Fortbestand der Leistungskategorien von stationär und ambulant festgehalten wird, solange bleibt auch der Entscheidungshorizont offen, entlang eines den Personen zugeschriebenen „stationären Hilfebedarfs" darüber zu entscheiden, welche (begrenzten) Wohnoptionen prinzipiell offen stehen. Dies stabilisiert und fördert möglicherweise Risiken der Diskriminierung und Separation von Personen mit hohen Unterstützungsbedarfen unter den finanziellen Vorzeichen der steigendenden Sozialhilfeausgaben in den nächsten Jahren.

Ebenfalls als widersprüchlich zu Artikel 19 erscheinen gelegentliche Praxen

- der „Fehlplatzierung“ junger Menschen mit Beeinträchtigungen in Alten- und Pflegeheimen ${ }^{37}$,

- des unfreiwilligen Auszugs alt werdender Menschen mit Beeinträchtigungen aus ihrer vertrauten Wohnumgebung (in Einrichtungen der Behindertenhilfe) in Alten- und Pflegeheime sowie

- der unfreiwilligen Auswärtsunterbringung von Bürgerinnen und Bürger eines Herkunftsgebietes in Einrichtungen anderer Regionen.

\section{Bewertungen und Wünsche aus Nutzerperspektive}

Eine qualitative Bewertung der Wohnsituation und hierauf bezogener Wahlfreiheiten ist im Lichte der Grundsätze von Selbstbestimmung und Partizipation letztlich nur aus der Perspektive der betroffenen Menschen möglich. Wie bewerten Menschen mit alltäglichen Unterstützungsbedarfen selbst ihre gegenwärtige Wohnsituation? Welche Wünsche und Bedürfnisse haben sie in Bezug auf ihre alltägliche Lebensführung, welche Unterstützung wünschen sie, und wie würden sie entscheiden, wenn sie reale Chancen hätten, ihren Aufenthaltsort zu wählen und zu entscheiden, wo und mit wem sie leben? Im Zuge der wachsenden Bedeutung partizipativer Forschung liegen inzwischen verschiedene Studien vor, welche die subjektiven Wahrnehmungen und Bewertungen der beeinträchtigten Menschen in den Vordergrund stellen. Diese zeigen - trotz unterschiedlicher Wohnformen, regionaler Kontexte und Altersgruppen der Befragten - hohe Übereinstimmungen zu zentralen Aspekten der wohnbezogenen Unterstützung. Im Folgenden sollen einige ausgewählte, im Kontext von Artikel 19 bedeutsame Ergebnisse skizziert werden. Dabei beziehe ich mich im Wesentlichen auf folgende drei Studien:

37 Drolshagen, „Was mir fehlt, ist ein Zuhause“. Fehlplatzierung jüngerer Behinderter in hessischen Altenhilfe-Einrichtungen, Berlin 2006. 


\begin{tabular}{|c|c|c|c|c|}
\hline & Erhebungsregion & Wohnformen & $\begin{array}{l}\text { Erhebungs- } \\
\text { methode }\end{array}$ & $\begin{array}{l}\text { Anzahl der } \\
\text { Befragten }\end{array}$ \\
\hline „Kundenstudie“‘38 & $\begin{array}{l}\text { Berlin (ausgewählte } \\
\text { Bezirke) }\end{array}$ & $\begin{array}{l}\text { Familie, ambulant betreutes } \\
\text { Wohnen, Wohnheime }\end{array}$ & Fragebögen & $\mathrm{N}=207$ \\
\hline $\begin{array}{l}\text { „Lebensqualität } \\
\text { aus Nutzersicht“‘39 }\end{array}$ & $\begin{array}{l}\text { NRW (Bielefeld), } \\
\text { Baden-Württemberg } \\
\text { (Nordbaden) }\end{array}$ & Wohnheime & Interviews & $\mathrm{N}=142$ \\
\hline „Wohnen inklusiv““40 & Baden-Württemberg & $\begin{array}{l}\text { Familie, Wohngruppe, } \\
\text { Wohnheim }\end{array}$ & Fragebögen & $\mathrm{N}=931$ \\
\hline
\end{tabular}

Zunächst fällt auf, dass sich etwa die Hälfte der befragten Menschen allgemein zufrieden mit der gegenwärtigen Wohnsituation äußert, und zwar unabhängig von der jeweiligen Wohnform. Dieser Befund lässt verschiedene Interpretationen zu:

- Die Wohnsituation entspricht tatsächlich den individuellen Bedürfnissen und Vorstellungen;

- es sind keine Alternativen bekannt. Verschiedene Befragungsergebnisse weisen darauf hin, dass viele Menschen mit Beeinträchtigungen und ihre Angehörigen nicht gut über alternative Wohnmöglichkeiten oder das persönliche Budget informiert sind;

- die grundsätzliche Zufriedenheit lässt sich aber auch mit dem aus der Forschung bekannten Zufriedenheitsparadoxon bzw. mit der „Theorie der Homöostase subjektiven Wohlbefindens“ des australischen Lebensqualitätsforschers Cummins erklären ${ }^{41}$. Demzufolge sind die meisten Menschen bestrebt, ein gewisses Niveau an Wohlbefinden unter variierenden (auch ungünstigen) Lebensbedingungen aufrecht zu erhalten und erreichen dies ggf. auch auf dem Wege resignativer Anpassung. Für diese Erklärung sprechen einzelne Äußerungen von befragten Menschen wie „am besten man lässt alles so wie es ist, sonst eckst du nur an“ oder „was soll ich schon ändern“42.

Trotz der allgemeinen Zufriedenheitswerte gibt jedoch mindestens jede zweite befragte Person Veränderungswünsche an mit der Perspektive, zukünftig anders wohnen zu wollen. Dies kann durchaus als Ausdruck dafür gedeutet werden, dass das Leben in den jeweiligen Wohnformen nicht den Bedürfnissen und Lebenszielen dieser Personen

38 Seifert, Kundenstudie, Bedarf an Dienstleistungen zur Unterstützung des Wohnens von Menschen mit Behinderung, Berlin 2010.

39 Schäfers, Lebensqualität aus Nutzersicht. Wie Menschen mit geistiger Behinderung ihre Lebenssituation beurteilen, Wiesbaden 2008.

40 Metzler/Rauscher, Wohnen inklusiv. Wohn- und Unterstützungsangebote für Menschen mit Behinderungen in Zukunft (Projektbericht), Stuttgart 2004.

41 Schäfers (Fußn. 39), S. 46.

42 Schäfers (Fußn. 39), S. 256. 
entspricht. Hinweise hierauf liefern teils drastische Äußerungen von Wohnheimbewohner/innen wie ,ich würde die Einrichtung abreißen“, „Anstalt schließen“ oder „dass ich hier rauskomme“43. Dabei zeigen einige Formulierungen der Befragten, „dass sie die Wohneinrichtungen als geschlossen und vom Gemeindeleben getrennten Lebensraum erleben, z.B. wenn sie Wünsche danach äußern „draußen leben“ oder ,außerhalb wohnen“ zu können“44. Die konkrete Kritik dieser Personen in betreuten Wohngemeinschaften oder Wohngruppen eines Heims richtet sich auch auf das Zusammenleben mit Menschen, die in der Regel nicht selbst ausgesucht wurden. Zwar wird das Leben in Gemeinschaft von einigen Personen durchaus geschätzt, allerdings wird die Frage ,gibt es Menschen in der Wohngruppe/Wohnung, mit denen Sie nicht gern zusammen wohnen?" von der Hälfte der befragten Personen in der Studie von Schäfers bejaht ${ }^{45}$. Auch in der Kundenstudie von Seifert steht das häufig konfliktreiche Zusammenleben in Gruppen bei den negativen Aspekten des Alltags an erster Stelle ${ }^{46}$. Diese Ergebnisse legen erhebliche Diskrepanzen offen zwischen der faktischen Wohnsituation beeinträchtigter Menschen und ihrem Recht, darüber zu entscheiden mit wem sie leben möchten (Art. 19 a) BRK).

Ein autonomes Leben nach eigenen Vorstellungen zu führen, bedeutet auch, den Alltag nach eigenem zeitlichem Rhythmus gestalten zu können. Diesbezüglich wünschen sich Wohnheimbewohner weniger Reglementierungen und mehr Handlungsspielräume; dies betrifft z.B. die Abschaffung von Badeplänen, abendlichen Rückkehrzeiten und die Möglichkeit, Freunde und Partner/innen abends besuchen zu dürfen ${ }^{47}$. Diese konkreten Beispiele zeigen, wie begrenzt Entscheidungsspielräume für die Gestaltung des Alltags in Einrichtungen im Vergleich zu kulturell üblichen Spielräumen nach wie vor sein können. Dabei scheint sich dass das Ausmaß an erlebter Selbstbestimmung und Wahlfreiheit in ambulanten Wohnformen deutlich von stationären Wohneinrichtungen zu unterscheiden und zugleich mit der Größe von Wohneinheiten zusammen zu hängen. So gaben in der Studie von Seifert fast alle befragten Personen, die in einer eigenen Wohnung leben, an, selbst über die Gestaltung des Alltags bestimmen zu können. Hingegen stimmten nur etwa die Hälfte der Wohnheimbewohner/innen dieser Aussage zu ${ }^{48}$. Auch bei Schäfers sehen fast die Hälfte der befragten Wohnheimbewohner/innen eher wenige Wahlfreiheiten (z.B. bei der Entscheidung darüber, welche Person zu welchem Zeitpunkt Hilfen erbringen); mit steigendem Hilfebedarf nehmen die erlebten Wahlfreiheiten ab. Zugleich äußern Personen in Wohnheimen ohne Wohngruppenbezug bzw. in

43 Schäfers (Fußn. 39), S. 330.

44 Schäfers (Fußn. 39), S. 330.

45 Schäfers (Fußn. 39), S. 254.

46 Seifert (Fußn. 38), S. 104.

47 Schäfers (Fußn. 39), S. 257.

48 Seifert (Fußn. 38), S. 106. 
sehr kleinen Gruppen (bis zu 3 Personen) deutlich höhere Zufriedenheitswerte mit Wahlmöglichkeiten als in größeren Wohngruppen ${ }^{49}$.

Wenn man vor diesem Hintergrund davon ausgeht, dass die Entscheidungsspielräume und Handlungsoptionen in ambulanten Wohnformen grundsätzlich größer sind, dann überrascht zunächst ein Ergebnis der Kundenstudie, demnach sich nur knapp die Hälfte der Menschen im betreuten Einzelwohnen zufrieden mit ihrer Wohnsituation äußert ${ }^{50}$. Die Kritik der Unzufriedenen bezieht sich jedoch nicht auf die Wohnform an sich, sondern auf die konkreten Wohnbedingungen, wie die Beschaffenheit der Wohnung und das (soziale) Wohnumfeld (z.B. kleine Wohnung, schlechte Infrastruktur, laute Nachbarn). Menschen, die alleine in einer Wohnung leben, äußern darüber hinaus zum Teil Einsamkeitsgefühle oder Probleme mit ihrem Partner. Im Bereich der sozialen Einbindung bestehen insgesamt Defizite in allen Wohnformen, dies bestätigen auch andere vorliegende Studien ${ }^{51}$. Vor allem Menschen mit kognitiven und psychischen Beeinträchtigungen in Wohneinrichtungen haben deutlich eingeschränkte informelle soziale Kontakte, insbesondere zu Personen außerhalb der Einrichtungen, und zugleich ein hohes Maß an nicht selbstgewählten Beziehungen, etwa zu Mitarbeiter/innen in den Einrichtungen und Diensten.

Gefragt nach ihren Wohnwünschen äußern die Personen insgesamt eine Vielfalt an Vorstellungen, wie und mit wem sie gerne wohnen und leben möchten: alleine, in Partnerschaft oder in einer Wohngemeinschaft mit Freunden, in der Familie usw. Diese Vielfalt der Wohnwünsche spiegelt allgemeine gesellschaftliche Tendenzen hin zu pluralen Wohn- und Lebensformen. Und die Ergebnisse unterstreichen die Unterschiedlichkeit von Menschen mit Beeinträchtigungen sowie die Notwendigkeit, von pauschalen Vorstellungen „behindertengerechter“ Wohnformen abzurücken. Am häufigsten wird in allen Studien das eigenständige Wohnen, alleine oder mit Partner/in, genannt. Hingegen spielen Wohnheime, Wohngemeinschaften und das Zusammenleben mit Angehörigen bei den Wohnwünschen der Personen mit Veränderungswünschen zahlenmäBig nur eine sehr nachrangige Rolle.

\section{Fazit und Perspektiven der Enthinderung}

Die Darstellung und Diskussion von Ergebnissen zur Wohnsituation von Menschen mit Beeinträchtigungen kann im Rahmen dieses Beitrages nur holzschnittartig bleiben. Insbesondere bleiben Kontextfaktoren im Sozialraum aufgrund der unzureichenden Da-

49 Schäfers (Fußn. 39), S. 332.

50 Seifert (Fußn. 38), S. 101.

51 Heckmann, Alltags- und Belastungsbewältigung und soziales Netzwerk, in: Beck/Greving (Hrsg.), Lebenslage, Lebensbewältigung. Enzyklopädisches Handbuch der Behindertenpädagogik Band 5, Stuttgart 2012, S. 115-123. 
tenlage ausgeblendet. Gleichwohl lassen bereits diese Ergebnisse im Zusammenhang mit den zuvor skizzierten Strukturdaten erhebliche Diskrepanzen zwischen den in Artikel 19 formulierten Ansprüchen auf eine selbstbestimmte Lebensführung und den faktischen Wahlmöglichkeiten im Kontext der organisierten wohnbezogenen Unterstützung erkennen. Die Vielfalt der Wohnwünsche mit einem hohen Stellenwert „einer eigenen Wohnung mit der individuell notwendigen Assistenz, in sozialen Bezügen und einem sozialen Umfeld, das eine gute Infrastruktur bietet" 52 einerseits und die zahlenmäßige Dominanz stationärer Einrichtungen, die finanziellen sowie rechtlich-administrativen Hürden bei der Umsetzung individuell passender Hilfen, einschließlich integrierter, trägerübergreifender Lösungen (beispielsweise im Verhältnis von Pflege und Eingliederungshilfe) andererseits klaffen weit auseinander. Das Recht zu entscheiden, wo und mit wem man leben möchte, lässt sich in diesem Rahmen ebenso unzureichend verwirklichen wie die Gestaltung eines Alltags nach eigenen Vorstellungen.

Vor diesem Hintergrund vollziehen sich bereits seit einigen Jahren wesentliche Schritte der Neuausrichtung von Wohnkonzepten für beeinträchtigte Menschen in Richtung personenzentrierter und gemeindeorientierter Unterstützung. Insbesondere mögliche Anpassungen der Eingliederungshilfe an politische, rechtliche, fachliche und gesamtgesellschaftliche Entwicklungen stehen gegenwärtig stark im Fokus ${ }^{53}$. Eine zentrale Rolle spielt im Rahmen dieser Bemühungen der konsequente Ausbau offener Hilfen. Allerdings zeigen die Ergebnisse zur Bewertung der Situation in ambulant betreuten Wohnformen, dass eine Umsteuerung von stationären zu ambulanten Hilfen allein nicht mehr Wohn- und Lebensqualität garantieren kann. Zwar ermöglicht das Leben in einer eigenen Wohnung strukturell ein hohes Maß an Selbstbestimmung und Wahlfreiheiten bei der Gestaltung des Alltags, es verhindert jedoch nicht per se Risiken sozialer Diskriminierung und der „Isolation und Absonderung von der Gemeinschaft“ (Art. 19 c BRK). Es bedarf vielmehr der individuellen Unterstützung bei der aktiven Herstellung und Aufrechterhaltung von Kontakten und Beziehungen im sozialen Nahraum, um eine Einbindung auch auf der Ebene sozialer Interaktion zu ermöglichen. Dies gilt umso mehr, als Menschen mit Beeinträchtigungen ohne Möglichkeiten informeller Unterstützung vollständig von professionellen, offenen Hilfen abhängig sind, die aber gegenwärtig in vielen Regionen eben nicht bedarfsgerecht verfügbar sind.

Ein zentraler Schlüssel für eine zielführende Neuausrichtung der sozialen Hilfen im Lichte von Artikel 19 BRK liegt in der Überwindung der Leistungskategorien ambulant und stationär und einer konsequenten Umstellung von einer Kategorie des „Wohnens“ auf Kategorien der Lebensführung bzw. der Lebens- und Alltagsbewältigung. „Wohnen" steht im System der traditionellen Behindertenhilfe für eine etablierte institutionelle Kategorie, die auf der rechtlich-administrativen Konstruktion eines pauschalen Hilfe-

52 Seifert (Fußn. 38), S. 21 f.

53 Vgl. Beschluss der 86. ASMK-Konferenz 2009 zur Weiterentwicklung der Eingliederungshilfe für Menschen mit Behinderungen („Eckpunkte für die Reformgesetzgebung Eingliederungshilfe im SGB XII“). 
bedarfs gründet, an den sich in der Regel, auf der Basis pauschaler Leistungs- und Vergütungsvereinbarungen zwischen Sozialhilfeträger und Anbieter, eine professionell organisierte Wohnform anschließt. Eine wirksame Unterstützung ist jedoch keine Frage nach dem richtigen Gebäude, oder anders formuliert: Das Wohnen ist kein Unterstützungsbedarf. Jede zielführende Intervention zur Teilhabe am Leben in der Gemeinschaft, die der Pluralisierung von Wohn- und Lebensformen und dem Postulat der Selbstbestimmung Rechnung tragen will, muss sich an der individuellen Lebensführung im Sinne des alltagspraktischen Zusammenhangs aller Aktivitäten einer Person in ihren verschiedenen konkreten Lebensbereichen ausrichten ${ }^{54}$. In diesem Betrachtungsrahmen gilt es danach zu fragen, wie Barrieren im Zugang zu sozial anerkannten Verwirklichungsräumen abgebaut, wie funktionale Beeinträchtigungen bewältigt und mit welchen Ressourcen der Alltag nach eigenen Vorstellungen gelingen kann. Diese Aufgaben lassen sich nicht mit pauschalen Maßnahmen im Sinne „behindertengerechter“ Wohnformen lösen, sondern sind höchst individuell in Abhängigkeit von persönlichen Voraussetzungen (Ressourcen, Fähigkeiten und Bedarfe/Bedürfnisse) und von konkreten regionalen und sozialen Bezügen am selbstgewählten Wohn- und Lebensort zu bearbeiten. Erforderlich ist daher eine enge Verknüpfung von Sozialraumentwicklung und der Weiterentwicklung personenzentrierter Hilfen bzw. ein optimiertes Zusammenspiel von örtlicher und regionaler Teilhabeplanung, die auf die Lebenslagen beeinträchtigter Menschen gerichtet ist ${ }^{55}$, mit Ansätzen individueller Hilfeplanung.

54 Zum soziologischen Konzept der alltäglichen Lebensführung vgl. Kudera/Voß (Hrsg.), Lebensführung und Gesellschaft, Opladen 2000.

55 Blankenfeld/Wachtel, Auf dem Weg ins Gemeinwesen - kommunale Praxis für Menschen mit Behinderung in Theorie und Praxis, VHN 2009, S. 197-211; Lampke/Rohrmann/Schädler, Örtliche Teilhabeplanung mit und für Menschen mit Behinderungen, Wiesbaden 2011. 


\section{Literatur}

Banafsche, Minou, Art. 19 Unabhängige Lebensführung und Einbeziehung in die Gemeinschaft, in: Welke, Antje (Hrsg.), UN-Behindertenrechtskonvention mit rechtlichen Erläuterungen. Deutscher Verein, Berlin 2012, S. 150-163.

BAGüS/con_sens, Kennzahlenvergleich der überörtlichen Träger der Sozialhilfe 2010, Hamburg 2011, http://www.consens-info.de/upload/files/projekte/z_projekte_dateien_

117219_ueoeTr_Benchmarking_Bericht_2010_Endfassung.pdf.

Bank-Mikkelsen, Niels, Denmark, in: Flynn, Robert/Nitsch, Cathreen (Hrsg.), Normalization, social integration, and community services, Baltimore 1980, S. 51-70.

Beck, Iris/Greving, Heinrich (Hrsg.), Lebenslage und Lebensbewältigung. Enzyklopädisches Handbuch der Behindertenpädagogik 5, Stuttgart 2012.

Beck, Iris/Greving, Heinrich, Lebenswelt, Lebenslage, in: dies. (Hrsg.), Lebenslage und Lebensbewältigung. Enzyklopädisches Handbuch der Behindertenpädagogik 5, Stuttgart 2012, S. 15-59.

Bielefeldt, Heiner, Zum Innovationspotenzial der UN-Behindertenrechtskonvention. Deutsches Institut für Menschenrechte. 3. Aufl. Berlin 2009.

Blankenfeld, Christine/Wachtel, Grit, Auf dem Weg ins Gemeinwesen - kommunale Praxis für Menschen mit Behinderung in Theorie und Praxis, VHN 2009, S. 197-211.

Burzan, Nicole et al., Das Publikum der Gesellschaft. Inklusionsverhältnisse und Inklusionsprofile in Deutschland, Wiesbaden 2008.

Drolshagen, Markus, „Was mir fehlt, ist ein Zuhause“. Fehlplatzierung jüngerer Behinderter in hessischen Altenhilfe-Einrichtungen, Berlin 2006.

Engels, Dietrich, Lebenslagen, in: Maelicke, Bernd (Hrsg.), Lexikon der Sozialwirtschaft, Baden-Baden 2008, S. 643-646.

Hahn, Martin, Behinderung als soziale Abhängigkeit. Zur Situation schwerbehinderter Menschen, München 1981.

Heckmann, Christoph, Alltags- und Belastungsbewältigung und soziales Netzwerk, in: Beck, Iris/Greving, Heinrich (Hrsg.), Lebenslage, Lebensbewältigung. Enzyklopädisches Handbuch der Behindertenpädagogik. Band 5, Stuttgart 2012, S. 115-123.

Hornberg, Claudia/Schröttle, Monika, Vorstudie zur Neukonzeption des Behindertenberichts, Bundesministerium für Arbeit und Soziales (Hrsg.), Forschungsbericht 408, 2011, http://www.bmas.de/DE/Service/Publikationen/Forschungsberichte/ForschungsberichteTeilhabe/fb-fb408-vorstudie-zur-neukonzeption-des-behindertenberichtes.html.

Kudera, Werner/Voß, Günther (Hrsg.), Lebensführung und Gesellschaft, Opladen 2000.

Lampke, Dorothea/Rohrmann, Albrecht/Schädler, Johannes, Örtliche Teilhabeplanung mit und für Menschen mit Behinderungen, Wiesbaden 2011.

Leßmann, Ortrud, Lebenslagen und Verwirklichungschancen (capability) - Verschiedene Wurzeln, ähnliche Konzepte, Vierteljahreshefte zur Wirtschaftsforschung 75/2006, 1, S. 30-42.

Luhmann, Niklas, Die Gesellschaft der Gesellschaft. Bd. 2, Frankfurt a.M. 1999. 
Rappaport, Julian, Ein Plädoyer für die Widersprüchlichkeit: Ein sozialpolitisches Konzept des „empowerment“ anstelle präventiver Ansätze, Verhaltenstherapie und psychosoziale Praxis $2 / 1985$, S. $257-278$.

Rudloff, Wilfried, Das Ende der Anstalt?, in: Bösl, Elsbeth/Klein, Anne/Waldschmidt, Anne (Hrsg.), Disability History. Konstruktionen von Behinderung in der Geschichte. Eine Einführung, Bielefeld 2010, S. 169-190.

Schäfers, Markus, Lebensqualität aus Nutzersicht. Wie Menschen mit geistiger Behinderung ihre Lebenssituation beurteilen, Wiesbaden 2008.

Schäfers, Markus/Wansing, Gudrun, Familienunterstützende Hilfen (FUH). Alternativen zum betreuten Wohnen für Menschen mit Behinderung, Münster 2009, http://www.lwl.org/ spur-download/fuh/fuh-bericht.pdf.

Schlebrowski, Dorothee, Starke Nutzer im Heim. Wirkung Persönlicher Budgets auf soziale Dienstleistungen, Wiesbaden 2009.

Seifert, Monika, Kundenstudie. Bedarf an Dienstleistungen zur Unterstützung des Wohnens von Menschen mit Behinderung, Berlin 2010.

Sen, Amartya, Ökonomie für den Menschen. Wege zur Gerechtigkeit und Solidarität in der Marktwirtschaft, München, Wien 2000.

Statistisches Bundesamt, Ergebnisse der Sozialhilfestatistik 2010, Wiesbaden 2012. Download unter: https:/www.destatis.de/DE/Publikationen/WirtschaftStatistik/Sozialleistungen/ ErgebnisseSozialhilfe2010_032012.pdf?_blob=publicationFile.

Thimm, Walter, Leben in Nachbarschaften. Hilfen für Menschen mit Behinderungen, Freiburg 1994.

Thimm, Walter, Tendenzen gemeinwesenorientierter Hilfen - Gesellschaftliche Ausrichtungen und fachliche Konsequenzen, in: ders. (Hrsg.), Das Normalisierungsprinzip. Ein Lesebuch zu Geschichte und Gegenwart eines Reformkonzeptes, Marburg 2005.

Waldschmidt, Anne, Selbstbestimmung als Konstruktion. Alltagstheorien behinderter Frauen und Männer, 2. korr. Auflage Wiesbaden 2012.

Wansing, Gudrun, Der Inklusionsbegriff in der Behindertenrechtskonvention, in: Welke, Antje (Hrsg.), Behindertenrechtskonvention und rechtliche Erläuterungen, Berlin 2012, S. 93103.

Wansing, Gudrun, Inklusion in einer exklusiven Gesellschaft - oder: wie der Arbeitsmarkt Teilhabe behindert, Behindertenpädagogik 4/2012, S. 381-396.

Wansing, Gudrun/Westphal, Manuela, Teilhabeforschung, Disability Studies und Migrationsforschung verbinden. Konzepte von Behinderung und Migration in der Forschung, Orientierung 1/2012, S. 12-15.

Welti, Felix, Kommentar zu $§ 9$ SGB IX, in: Lachwitz, Klaus/Schellhorn, Walter/Welti, Felix (Hrsg.), HK-SGB IX, 3. Aufl. Köln 2010.

WHO, World Report on Disability, Genf 2011. 


\title{
Rechtliche Grundlagen einer örtlichen Teilhabeplanung ${ }^{1}$
}

\author{
Felix Welti
}

$\begin{array}{ll}\text { I. Einführung } & 87\end{array}$

$\begin{array}{lr}\text { II. Pflicht zur Teilhabeplanung? } & 89\end{array}$

1. Verfassungsrecht 90

2. Behindertenrechtskonvention 90

3. Bundes- und Landesgesetze 92

III. Teilhabeplanung durch Städte, Gemeinden und Kreise 93

1. Kommunale Selbstverwaltung 93

2. Kommunen als Träger staatlicher Aufgaben 99

3. Beteiligung behinderter Menschen 95

IV. Regionale Teilhabeplanung durch Sozialleistungsträger 96

1. Planungsverantwortung nach SGB I und SGB X 96

2. Planungsverantwortung für Leistungen zur Teilhabe nach SGB IX 97

3. Planungsverantwortung für Pflegeleistungen nach SGB XI 98

4. Gesundheitsplanung 99

$\begin{array}{ll}\text { V. Schluss } & 100\end{array}$

\section{Einführung}

Die Aktivitäten behinderter Menschen und ihrer Verbände für mehr Selbstbestimmung und Teilhabe, die neuere wissenschaftliche und fachliche Diskussion, insbesondere über die Internationale Klassifikation der Behinderung, Funktionsfähigkeit und Gesundheit $(\mathrm{ICF})^{2}$, und die Gesetzgebung mit SGB IX, Behindertengleichstellungsgeset-

1 Frühere Fassungen dieses Textes sind erschienen in ZfSH/SGB 2011, S. 401-406, und in Dorothea Lampke/Albrecht Rohrmann/Johannes Schädler (Hrsg.), Örtliche Teilhabeplanung mit und für Menschen mit Behinderungen, Wiesbaden 2011, S. 55-67.

2 Zum Zusammenhang zwischen der International Classification of Functioning, Disability and Health (ICF) der WHO und dem SGB IX auch Wolfgang Seger/Hans-Martin Schian/Bernd Steinke/Wolfgang Heipertz/Michael Schuntermann, Gesundheits-, sozial-, gesellschafts- und organisationspolitische Auswirkungen der Anwendung der ICF auf eine integrierte Rehabilitation, Das Gesundheitswesen 2004, S. 393-399. 